\title{
Development, Qualification and Integration of the Optical Fiber Array Assemblies for the Lunar Reconnaissance Orbiter.
}

\author{
Melanie N. Ott ${ }^{\mathrm{a}}$, Robert Switzer ${ }^{\mathrm{b}}$, Richard Chuska ${ }^{\mathrm{b}}$, \\ Frank LaRocca ${ }^{\mathrm{b}}$, William Joe Thomas ${ }^{\mathrm{b}}$, Shawn Macmurphy ${ }^{\mathrm{b}}$ \\ ${ }^{a}$ NASA Goddard Space Flight Center, Greenbelt Maryland, 20771 \\ ${ }^{b}$ MEI Technologies, 7404 Executive Place, Suite 500, Seabrook, MD 20706
}

\begin{abstract}
The NASA Goddard Fiber Optics Team in the Electrical Engineering Division of the Applied Engineering and Technology Directorate, designed, developed and integrated the space flight optical fiber array hardware for the Lunar Reconnaissance Orbiter (LRO). The two new assemblies that were designed and manufacturing at GSFC for the LRO exist in configurations that are unique in the world for the application of ranging and LIDAR. Described here is an account of the journey and the lessons learned from design to integration for the Lunar Orbiter Laser Altimeter and the Laser Ranging Application on the LRO.
\end{abstract}

\section{INTRODUCTION}

\subsection{The Lunar Reconnaissance Orbiter}

The LRO is the first in a series of missions towards the exploration of the moon and mars for the purpose of providing remote human habitat bases for planetary study. LRO will do extensive mapping of the moon surface in search of the most appropriate landing sites identify resources and explore the environment. High precision mapping of the moon is a requirement for any future moon landings. Dr. Farzin Amzajerdian of NASA Langley Research center points out in his recent article published in Photonics Spectra, "Even though Apollo missions targeted well-surveyed landing sites, they still experienced some close calls." In fact on page 55 of this July 2008 issue Dr. Amzajerdian illustrates just how close the Apollo 14 came to a small crater only inches away from one of its support structure legs. Therefore the goal of the Lunar Orbiter Laser Altimeter an instrument on the LRO will provide a 3 dimensional mapping of the lunar surface topography for accurate and safe location of landing sites.

\subsection{The Longest Laser Link in Space}

During the conceptual design of the LOLA instrument, an experiment with the Mercury Laser Altimeter or MLA in May 2005, that had launched in 2004 provided the assurance that laser communications could traverse long distances through space. A laser system built and designed at GSFC called "HOMER" [3] located at NASA's Goddard's Geophysical and Astronomical Observatory was used to contact the MLA aboard MESSENGER on its way to mercury and established a ranging link across 24 million kilometers. [4 -5].

\subsection{The Lunar Orbiter Laser Altimeter and Laser Ranging}

The experimental results were so promising that the Principal Investigators Dave Smith and Maria Zuber decided that LOLA would not only perform topography mapping of the moon but could also simultaneously make ranging measurements from the earth. Firing a $532 \mathrm{~nm}$ earth based laser from the station at Goddard's facility to the instrument LOLA could provide for high precision distance information using the timing of firing to receiving at the LOLA instrument. The challenge was to receive the laser pulses from the $532 \mathrm{~nm}$ Laser Ranging Telescope (LRT) located on the High Gain Antenna System located on the end of a boom arm pointed at the earth and transmit the information across 3 subsystems to one of the LOLA detectors in a compact but multi optical fiber configuration. The challenge of the LOLA instrument system was to reduce the size and weight of the previous MLA hardware design from four telescopes into one telescope with a fiber based array in a precise compressed pattern into a single connector.

\footnotetext{
${ }^{1}$ Melanie.n.ott@nasa.gov, 001-301-286-0127, misspiggy.gsfc.nasa.gov/photonics
} 

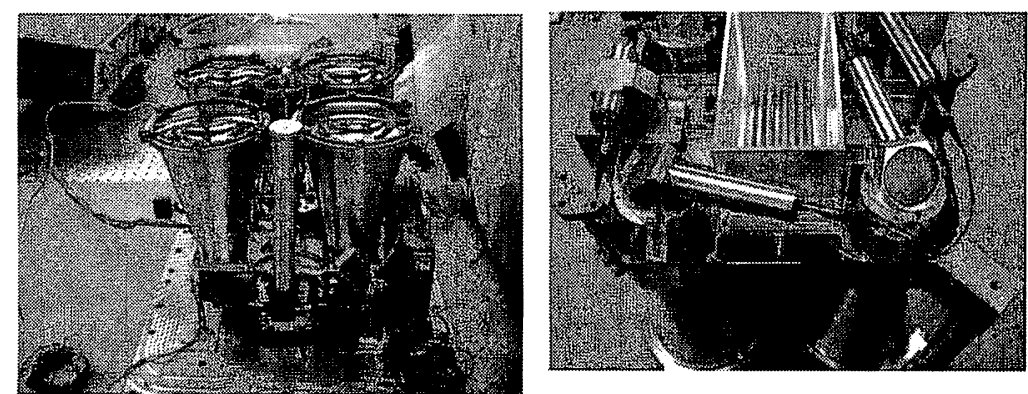

Figure 1: The Mercury Laser Altimeter consisted of four telescopes with fiber coupled form each to four individual detectors.[6]

Where the Mercury Laser Altimeter consisted of four telescopes and is designed to be capable of measuring with a $0.5 \mathrm{~m}$ precision, LOLA will consist of only one telescope and provide a 0.1 meter precision. Both were designed to function using pulsed $1064 \mathrm{~nm}$ lasers and measuring the timing of the reflections with short duration pulses. LOLA uses a diffractive optical element to split a single laser beam into five separate pulsed beams with five very precisely positioned optical fibers behind the receiver optics to link each beam to its designated detector. The time of flight measurements provide ranging information, the pulse spreading corresponds to surface roughness, and the return energy provides surface reflectance. The five spots will provide slopes along and across the orbit track. [1] In addition, one of LOLA's detectors will be used to monitor the ranging signals received by the $532 \mathrm{~nm}$ pulsed laser from the Greenbelt facility. Figure 2 shows an artists rendition of the space craft configuration.
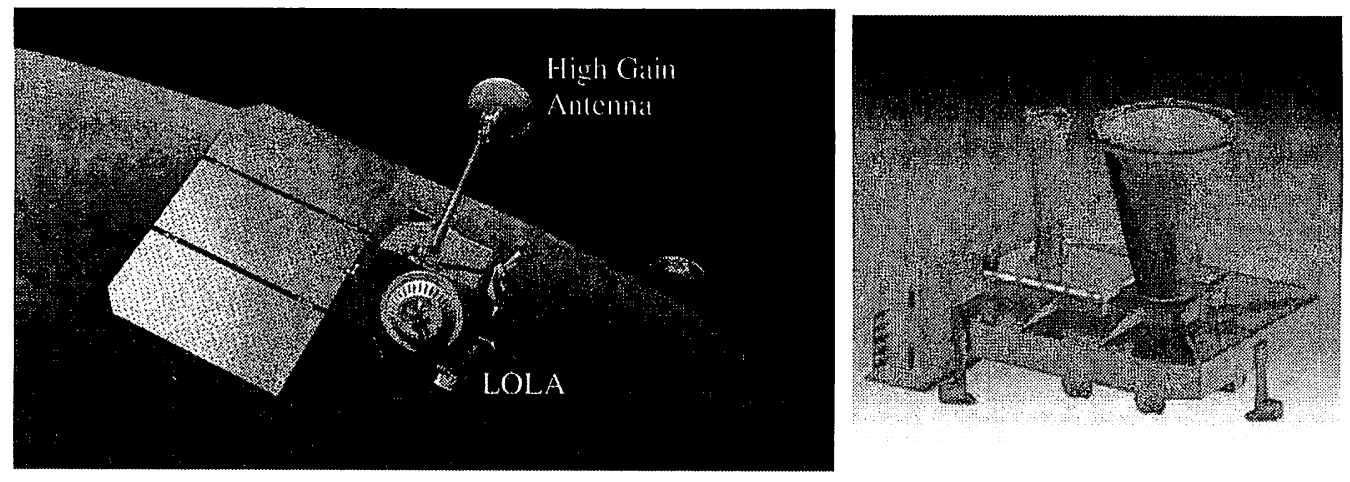

Figure 2: a) Artistic rendition of the LRO with the High Gain Antenna pointing at the earth while instrument LOLA is viewing the moon's surface, $b$ ) Artist rendition of LOLA.

\section{DISCUSSION}

For each of the ranging and lidar applications, the requirements were such that a single array connector would be required to perform the light collection from behind each of the receiver telescopes and early on a modified Diamond AVIM connector was the connector of choice due to its long standing space flight heritage that had started with Locheed Martin in the 1990's for single mode applications. Since then the Diamond AVIM had been flown on the ICESAT Geoscience Laser Altimeter system (GLAS), the Shuttle-Return-to-Flight high definition heat tile sensor camera, and the Mercury Laser Altimeter. The only challenge would be the fiber size chosen. The 200/220 micron step index FI series optical fiber was chosen to meet the optical performance requirements for LOLA, and the 400/440 micron step index FV series optical fiber produced by Polymicro Technologies was chosen for the Laser Ranging Application. For LOLA the requirements dictated 5 optical fibers held into very precise alignment with tight tolerancing and for LR the application required 7 fibers for optimal optical transmission while minimizing the termination challenges.

\subsection{LOLA Optical Assembly Design}


The solution to the LOLA challenge is pictured in Figure 3, a five fiber array on side A fanned out to individual standard AVIM connectors on side B. The most difficulty proved to be the pattern on the array ferrule. The holes had to be precisely aligned and the tolerancing tight but not too tight to disallow termination. That is not to say that the termination process was easy, it was indeed not. The process was arduous and time consuming with a $50 \%$ yield at best once all tests and polishing procedures were performed. No voids in the epoxy or defects of any kind would be acceptable for these very specialized space flight assemblies. The ferrules themselves were bought as blanks from Diamond USA/Switzerland and custom drilled in the NASA GSFC Mechanical Systems Engineering Division, Code 540.
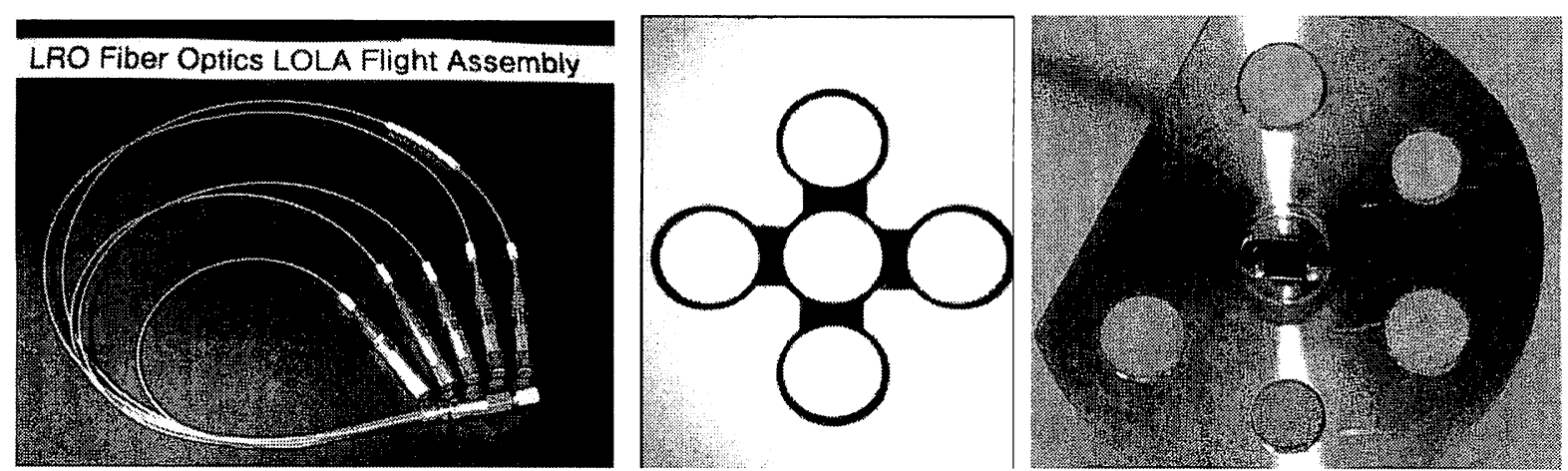

Figure 3 a) LOLA optical fiber array to fan out assembly, b) 200X magnification end face image of optical fiber array, c) the LOLA custom adapter interface to the receiver optics.

In addition to having difficult array fiber positioning requirements, the LOLA assembly also had very precise length requirements. Each fiber on the fan out side would require a different path to its designated LOLA detector and the detectors were spread around the instrument perimeter. Both for Laser Ranging and LOLA applications a larger diameter AVIM connector would be required for ease of termination and long term reliability of the array termination strain relief. The spring needed to be enlarged to accommodate the larger core fibers with acrylate coating. Acrylate coatings are preferred due to the ease of removal with methylene chloride. Fiber optics for flight are best stripped with chemicals for longer term reliability. Any scoring or scratching due to tooling could result in latent failure modes that can not be fixed once the space instrument is launched.

\subsection{LOLA Manufacturing and Qualification}

The termination procedures used were screened with rigorous requirements validation with insitu measurements during survival level environmental testing. The screening details and results are in reference 9. One of the significant lessons learned was related to the vibration levels used for the requirements validation. Early on, the only available information regarding the system level vibration expectations was through knowledge of the launch vehicle and the appropriate profile as chosen by GEVS[10]. Once the flight hardware was built, it was requested by the instrument team that protoflight testing; meaning, testing on the actual flight articles prior to delivery to the instrument, would be required. In past history, most flight hardware was only tested for thermal workmanship levels but due to the nature of the new hardware design and packaging of the array assembly, a vibration test and an extended thermal workmanship test more typical of optical bulk subsystems were added for all flight articles. At the LOLA instrument level vibration and thermal vacuum testing were conducted. Once integrated the entire LRO space craft endured vibration and thermal. So the LOLA integration flight assembly as a protoflight article will have endured three vibration tests and three thermal vacuum qualification tests prior to launch delivery of LRO.

During manufacturing, screening and qualification the lessons learned were drafted into a flow and list of documents for quality controlling the repeatability of the product production. The flight delivery flow is in Figure 4. 


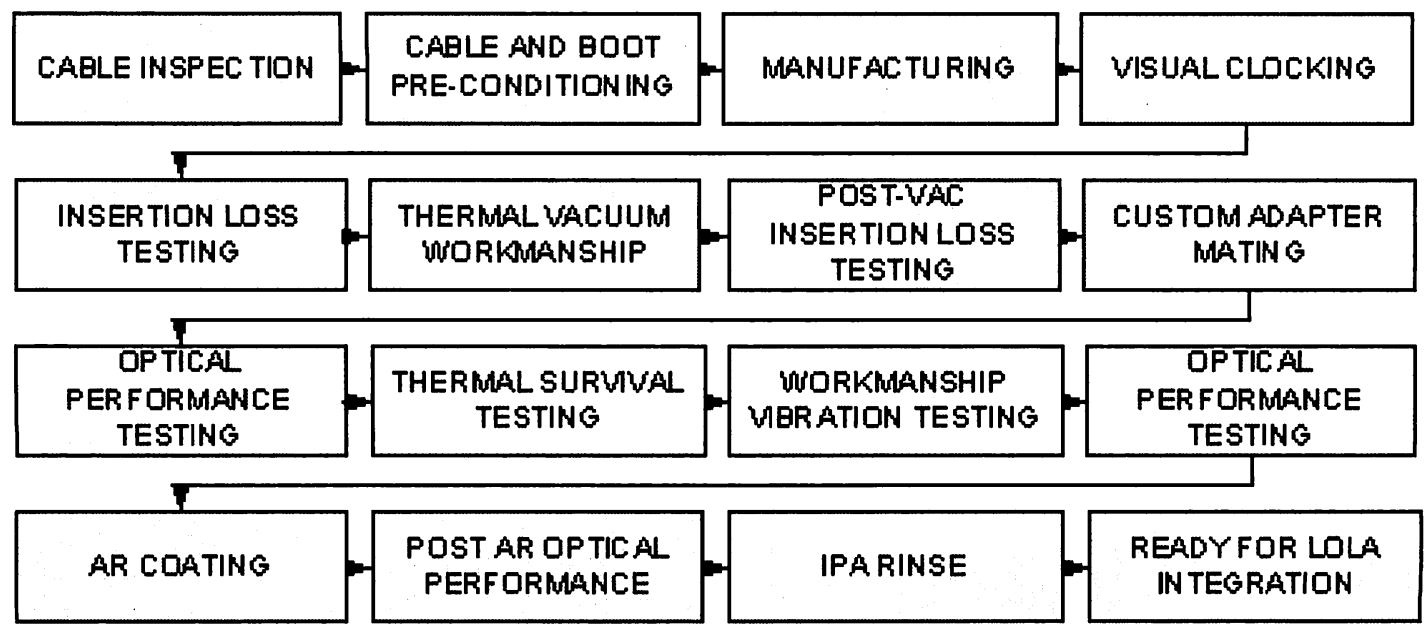

Figure 4: LOLA Optical Fiber Bundle Array Assembly Delivery Flow

As is common practice with all flight assemblies [6] all cables made of fluoropolymers and all connector boots must pass ASTM-E595 as an initial screen and are put through thermal preconditioning. For the hytrel boots on the LOLA assemblies, the preconditioning involves a high temperature vacuum bake out to ensure compliance to ASTM E595 post processing, and for cables the preconditioning procedure involves thermal cycling in ambient with hour long dwell soaks at the hot extremes and a half an hour at a cold temperature $\sim-30 \mathrm{C}$. The survival upper limit requirement for the subsystem is used as the hot thermal extreme during thermal preconditioning and the cycling is ramped at 1 to 2 degrees $\mathrm{C}$ /minute for anywhere between 40 to 70 cycles depending on the cable type. For LOLA the preconditioning was conducted for 50 cycles using the upper survival limit.[9] Assembly pieces are cut to approximately the length that will be used to maximize the effectiveness of the thermal preconditioning procedure.

\subsection{LOLA Bundle Fiber Assembly Quality Documentation}

The LOLA bundle assembly included a special mechanical adapter to coupled the light from the receiver optics to the bundle array. It was imperative that the clocking be exact such that the reflected five beam image from the surface of the moon align with the highest level of transmission possible. The clocking of the array into the adapter was performed visually under a microscope at $200 \mathrm{X}$ magnification such that mating to the adapter would be simple. The adapter held the array in a precise position of 25 microns from the end of the adapter interface to the receiver optics. The flight adapters ended up on the small side of the dimensional tolerance which caused the adapter to fit too tightly. Anomaly investigations were performed and proved that the stainless steel ferrules were causing metallic burrs on the tightly tolerance ferrule holes and this resulted in misalignment of the pattern along the optical axis. Once the assemblies were properly mated to the adapters they were not removed due to this anomaly. Rigorous processes were put in place to ensure the safety of the valuable flight hardware. The array strain relief was investigated for integrity and a compression test was added to the manufacturing procedures to ensure the quality of each array assembly that would not be found to be faulty by thermal vacuum workmanship testing alone. Figure 5 includes the list of documentation to ensure the quality of the manufacturing, testing and integration of the LOLA bundle array assemblies. 
Table 1: LOLA Documentation for Configuration Management

\begin{tabular}{|l|l|}
\hline Document Name & CM Documentation Number \\
\hline LOLA Fiber Optic Flight Assemblies & LOLA-OPTICS-WOA-0338 \\
\hline Thermal pre-conditioning on Flexlite 200/220 $\mu \mathrm{m}$ fibers for flight application & 562-PHOT-WI-LOLA-TP-001 \\
\hline Preconditioning Procedure for AVIM Hytrel Boots for LOLA fiber optic assemblies & 562-PHOT-WI-LOLA-VAC-001 \\
\hline Procedure for Diamond AVIMS PM Kit Pre-Assemble Inspection & LOLA-PROC-0104 \\
\hline $\begin{array}{l}\text { Assembly and Termination Procedure for the Lunar Orbiter Laser Altimeter Five Fiber } \\
\text { Custom PM Diamond@ AVIM Array Connector for the Lunar Reconnaissance Orbiter }\end{array}$ & LOLA-PROC-0098 \\
\hline $\begin{array}{l}\text { Insertion Loss Measurement Procedure For LOLA 5-Fiber Assembly (Open Beam } \\
\text { Configuration) }\end{array}$ & 562-PHOT-WI-LOLA-IL-001 \\
\hline Integration of the LOLA Fiber Optic Bundle to the Telescope Adapter & LOLA-PROC-0140 \\
\hline LOLA Fiber Bundle Inspection and Test Procedure & LOLA-PROC-0099 \\
\hline
\end{tabular}

Once all testing was performed and the assemblies accepted for flight, they were rinsed in isopropyl alcohol to reduce the contamination of the assembly to other parts of the flight instrument. Contamination for LOLA was a high profile issue due to the location of the instrument on LRO. Nearby would be an instrument that would be functioning in the UV where contamination is much more of a problem for the optical systems in that wavelength range due to the darkening of residue that outgases from other objects and collects on smooth optical surfaces. This is the single most common failure mode among laser systems that have many optical interfaces.[10]

\subsection{LOLA Optical Fiber Bundle Array Integration.}

Several fit checks were performed on the LOLA engineering mock up such that flight integration time came, the team would be sure that the flight hardware would fit exactly to the flight configuration. Integration of the flight hardware occurred in October of 2007. The pictures in Figure 6 show the integration of the flight optical fiber bundle array assembly to the LOLA flight instrument.
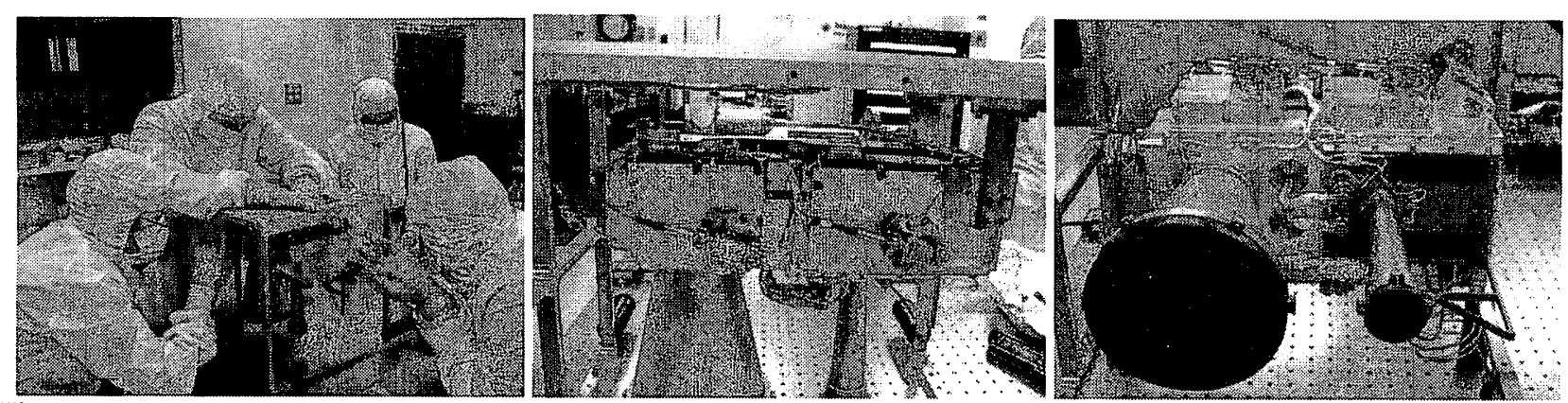

Figure 6 a) LOLA flight integration team including lead mechanical engineer, Steve Schmidt, lead fiber optics engineer Melanie Ott, Quality Assurance Engineer Keith Cleveland and Optical Engineer, Robert Switzer, b) side view of two detectors that were coupled to two channels of the fan out side of the optical bundle assembly, c) LOLA instrument fiber integration complete.

\subsection{The Laser Ranging Application}

The Laser Ranging application proved much more challenging than the LOLA optical fiber bundles. The main reason was due to the necessity for traversing 3 subsystems which included, the boom arm of the High Gain Antenna System, the HGAS gimbals used to steer the antenna, and the long run across the space craft over to the LOLA detector on the 
opposite end of the space craft. For ease of integration, it was necessary for three cables to complete this set which implied two optical fiber array to array interconnections of precise clocked alignment.

\section{LR Operations Overview}

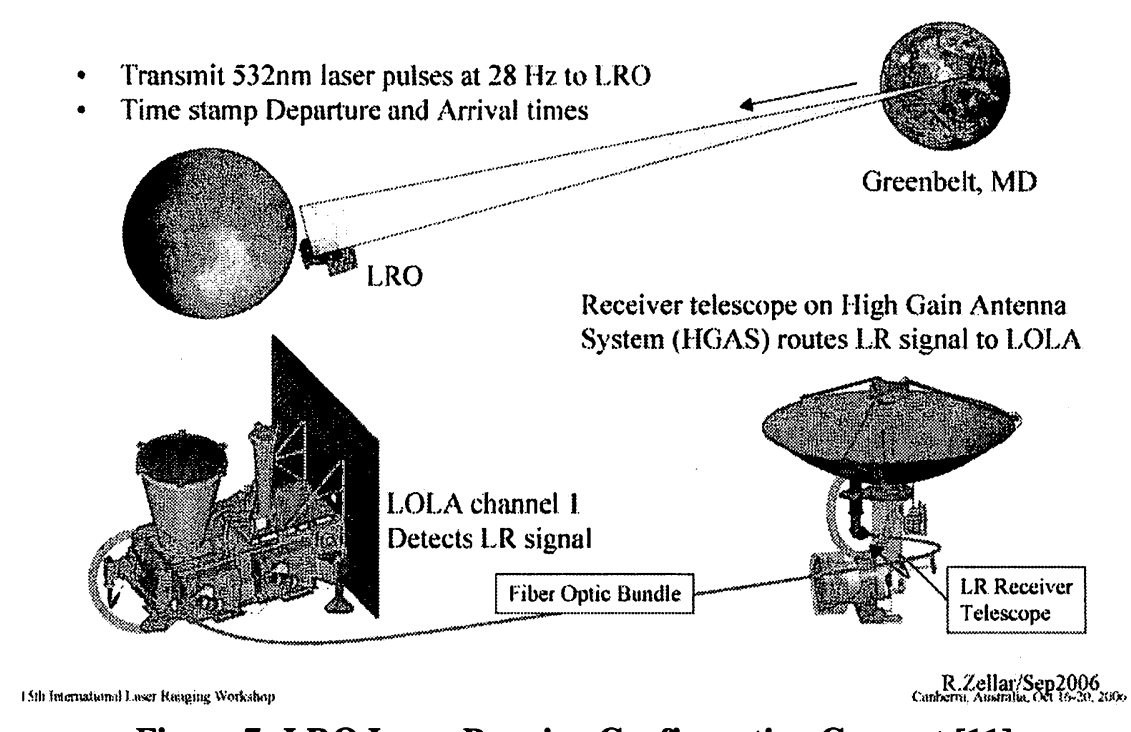

Figure 7: LRO Laser Ranging Configuration Concept [11]

Figure 7 shows the concept as drafted by System Instrument Manager Ronald Zellar during Engineering Peer Reviews for Laser Ranging on LRO and presented at the Laser Ranging Workshop by Dave Smith in 2006. The signal from the earth is received by the Laser ranging telescope and routed over from the High Gain Antenna, through the gimbals, down the boom, around a deployment mandrel and over to the LOLA detector channel 1.

\section{LR Flight System Components}

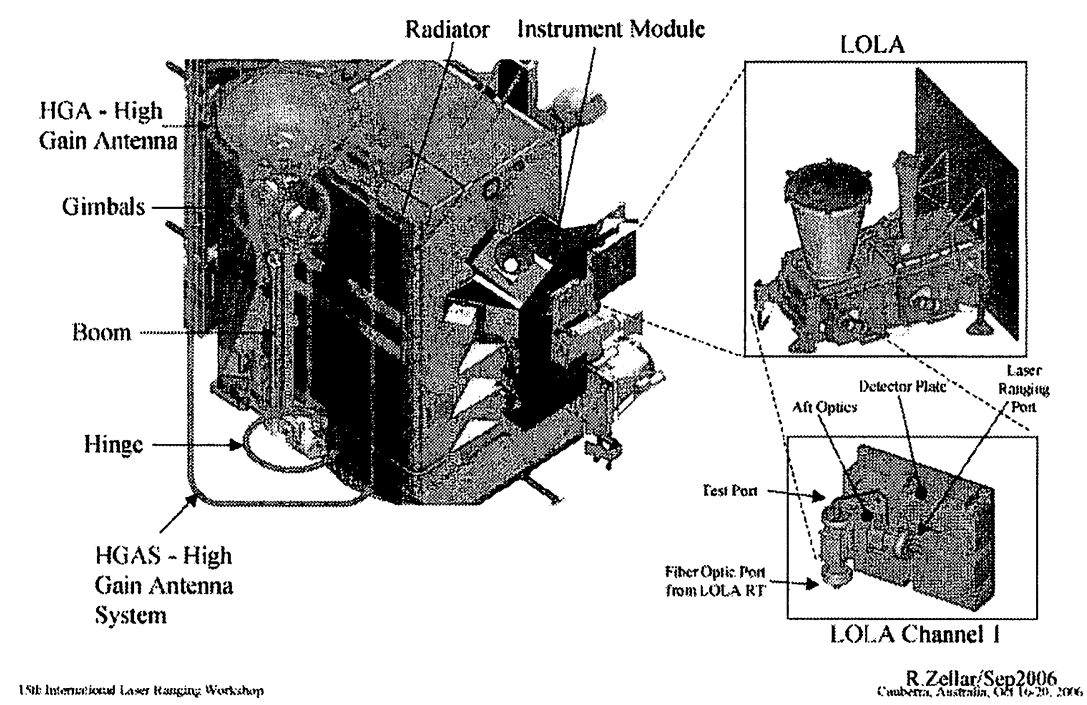

Figure 8: Details on the LRO Optical Fiber Link Path from the LRT to LOLA, D. Smith [12]

In Figure 8 the complexity of the fiber route is represented with greater detail. Since the subsystems would have to be assembled at various times during the integration process, the requirement was set early on to have an interconnection point between gimbals 1 and 2 and another where the entire High Gain Antenna System linked to the space craft on LRO. 


\subsection{Development of Laser Ranging Application Optical Fiber Bundle Array Assemblies}

The Laser Ranging assemblies were designed for enhanced reliability, flexibility and stable performance over a very wide thermal range. Originally the thermal requirement for the cold limit was $-75 \mathrm{C}$. Couple the cold thermal extreme with all the motion expected from the coiling and uncoiling that occurs inside of the gimbal cable wraps and the deployment hinge and its clear that to couple enough light reliably would require more than fiber. The optics team chose 7 optical fibers at $400 / 440$ microns to provide optimal optical performance while maintaining mechanical flexibility. The ferrules were then an issue because again as with LOLA, the pattern for the fibers had to be precisely centered and within a tight tolerance to be mated to another assembly.

The largest challenge the team faced was to go from concept to flight hardware in less than two years. The AVIM connector had to have clocking ability as with FC type polarization maintaining connectors and be enlarged due to the size of the fiber with its acrylate coating. Diamond Switzerland along with the Mechanical Systems Division efforts lead by Adam Matuzseski at Goddard Space Flight Center worked the challenges of making the new customized PM AVIM successful. During EM prototyping the ferrule material went from stainless steel 303 to stainless steel 416 to allow for the larger thermal excursion. Neither of these material types were used with the commercial AVIM connectors but for ease of the fiber pattern drilling it was the necessary choice. The custom drilling of the ferrules were performed both by NASA Goddard and by Diamond Switzerland. For the flight models the Diamond Switzerland ferrules were used.
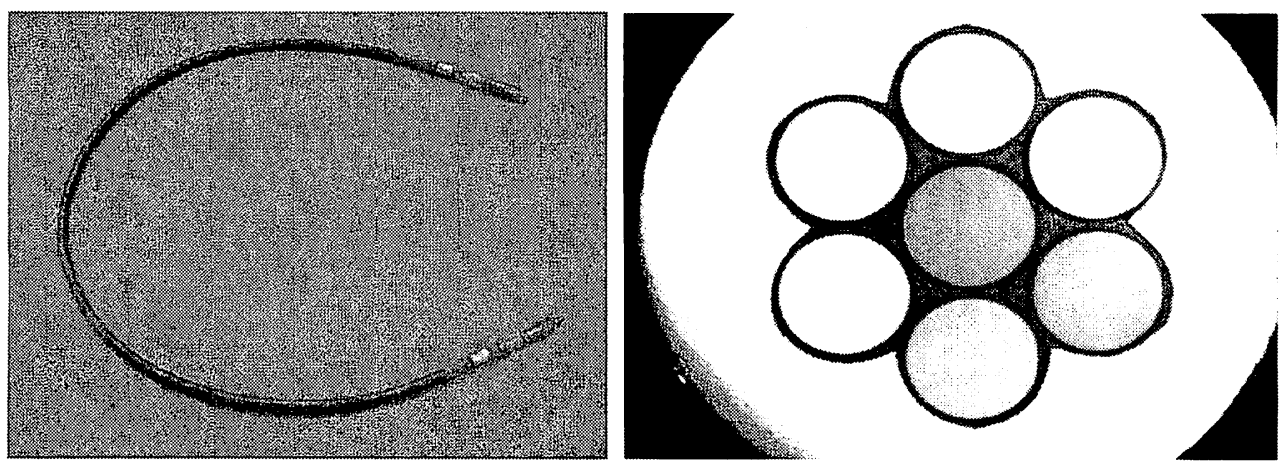

Figure 9 a) Example of a LR seven fiber optical assembly, b) endface picture of Diamond ferrule at $200 X$ magnification with 7 440 micron outer diameter optical fibers packed precisely together to minimize the epoxy bond line.

The optical requirements were difficult to meet since the losses at the interfaces were complicated by the endface concave polishing such that the arrays would not be in physical contact such that real time optimization could be performed on the actual adapters that would be used for flight if need be. The actual adapters used to perform clocking optimization were purposely gapped to avoid any issues with contamination causing gouging or cracking of the optical fiber array during this process. Originally the low profile standard AVIM adapter was chosen but after mechanical difficulties similar to those experienced on LOLA, the Diamond "Cleanable" adapter was used for its ceramic insert that did not experience the scoring that was experienced using a stainless ferrule with the low profile adapter.

The bundle assembly was made to fit the existing gimbal design such that no risk would result from putting an optical fiber harness in the gimbal systems as opposed to the heritage metallic wire harnessing. Considerable effort went into designing the engineering model assembles to avoid none failure modes and tested under insitu monitoring conditions for:

- Array Compression Testing.

- Thermal Vacuum Workmanship Testing, 8 cycles

- Vibration Launch Conditions.

- Thermal Cycling with Active Monitoring (accelerated life)

- Cold Gimbal Motion Testing, 20,000 Mechanical Cycles with Active Monitoring

- Gimbal Life Testing, 20,000 Motion Cycles.

- Gamma Radiation Testing with Active Monitoring.

Reference 13 contains the bulk of the data collected on the engineering models that paved the way for the documentation list for the flight bundle assemblies. The list of quality documentation is listed in Table 2. 
Table 2: Laser Ranging Procedures and Quality Documentation

\begin{tabular}{|l|c|}
\hline Document Title & Document Number \\
\hline Thermal Pre-conditioning on Flexlite 200/220 $\mu \mathrm{m}$ fibers for flight application & LOLA-PROC-0137 \\
\hline Preconditioning Procedure for AVIM Hytrel Boots for LOLA fiber optic assemblies & LOLA-PROC-0138 \\
\hline Diamond AVIM PM Kit Pre-Assembly Inspection & LOLA-PROC-0104 \\
\hline Ferrule Polishing \& Ferrule/Adapter Matching Procedure & LOLA-PROC-0139 \\
\hline $\begin{array}{l}\text { Assembly and Termination Procedure for the Laser Ranging Seven Fiber Custom PM Diamond AVIM } \\
\text { Array Connector for the Lunar Reconnaissance Orbiter }\end{array}$ & LOLA-PROC-0112 \\
\hline Compression Test Procedure for Fiber Optic Connector & LOLA-PROC-0141 \\
\hline Active Optical Power Optimization Procedure for The Laser Ranging Optical Fiber Array Assemblies & LOLA-PROC-0110 \\
\hline Laser Ranging Fiber-Optic Bundle Optical Test Procedure & LOLA-PROC-0107 \\
\hline Insertion Loss Measurement Procedure for The Laser Ranging Optical Fiber Array Bundle Assemblies & LOLA-PROC-0111 \\
\hline Mating of Two LR 7-Fiber Optical Fibers Using Cleanable Adapter & LOLA-PROC-0142 \\
\hline Cutting Back The Kynar Strain Relief For Integration & LOLA-PROC-0143 \\
\hline Fiber Optic Bundle Inspection and Insertion Loss Measurement & LOLA-PROC-0148 \\
\hline
\end{tabular}

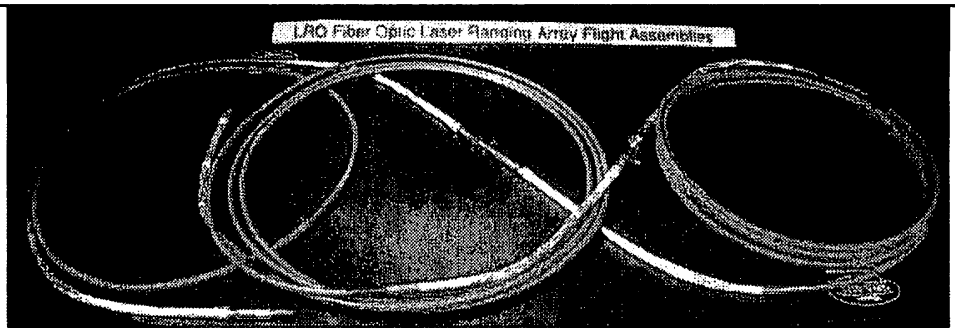

Figure 10: Flight Array Bundle Assembly Set

Several anomalies were investigated during the course of the development from the engineering models to the flight models and as a result the jacketing was removed from immediate contact with the connector. Figure 10 shows the final configuration of the Laser Ranging cable set that was approximately $10 \mathrm{~m}$ in length.

\subsection{Integration of the Laser Ranging Assemblies}

Integration took approximately 7 months and was challenging. Some subsystems were being assembled simultaneously which made having the necessary resources on hand to accomplish the testing at each location a difficult task. The optical fiber assemblies were arranged as such

Table 3: Fiber Optic Bundle (FOB) designations and integration locations.

\begin{tabular}{|l|l|}
\hline $\begin{array}{l}\text { Optical } \\
\text { Assembly }\end{array}$ & Subsystem Link \\
\hline FOB 1 & Laser Ranging Telescope, through Gimbal 1, to the bracket between Gimbals 1 and 2. \\
\hline FOB 2 & Bracket between Gimbals 1 \& 2, through Gimbal 2, down the boom, and around the deployment mandrel. \\
\hline FOB 3 & Bracket for the High Gain Antenna System to Detector 1 on LOLA. \\
\hline
\end{tabular}

The Laser Ranging system was integrated in the following order:

1) FOB 2 integrated into Gimbal 2 cable wrap and looped onto boom section.

2) FOB 1 integrated into Gimbal 1 cable wrap.

3) FOB $1 \& 2$ interconnected with gimbals 1 and 2 were combined, partial boom segment.

4) FOB 2 integrated completely to completed boom, and deployment mandrel.

5) Gimbals/Boom integrated to the High Gain Antenna.

6) LR telescope integrated to High Gain Antenna and connected to FOB 1.

7) FOB 3 integrated to Instrument Panel, extra looped aside in preparation for LOLA integration.

8) FOB 3 integrated to other side of LRO space craft.

9) LOLA integration.

10) FOB 3 interconnected to LOLA.

11) HGAS integrated to LRO space craft

12) FOB 3 interconnected to FOB 2 at LRO space craft bracket. 
Before and after every operation transmission tests were measured and monitored against the system level allocations. Motion testing was run insitu to monitor performance in the cable wraps of each gimbal during integration. Any exposed cables were covered with conducting tape for thermal control.
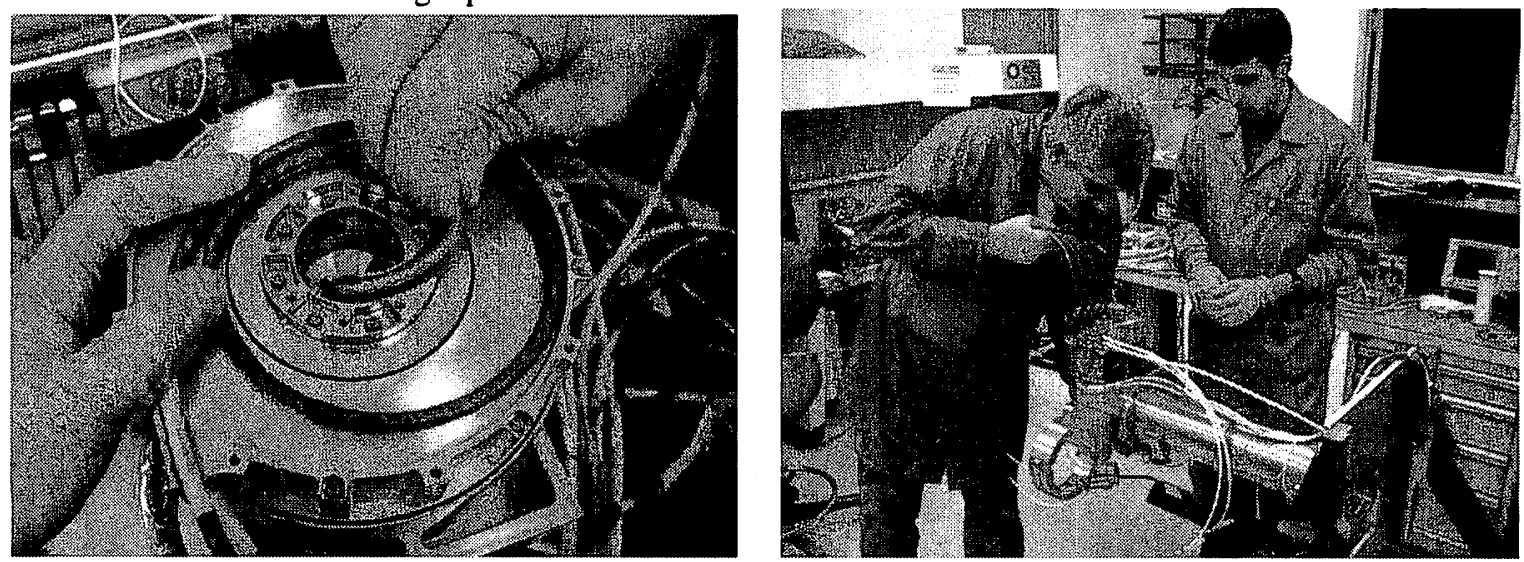

Figures 11 a) FOB 2 being routed into the cable wrap on gimbal 2, b) Photonics Group Members, Richard Chuska and Robert Switzer work on integration o
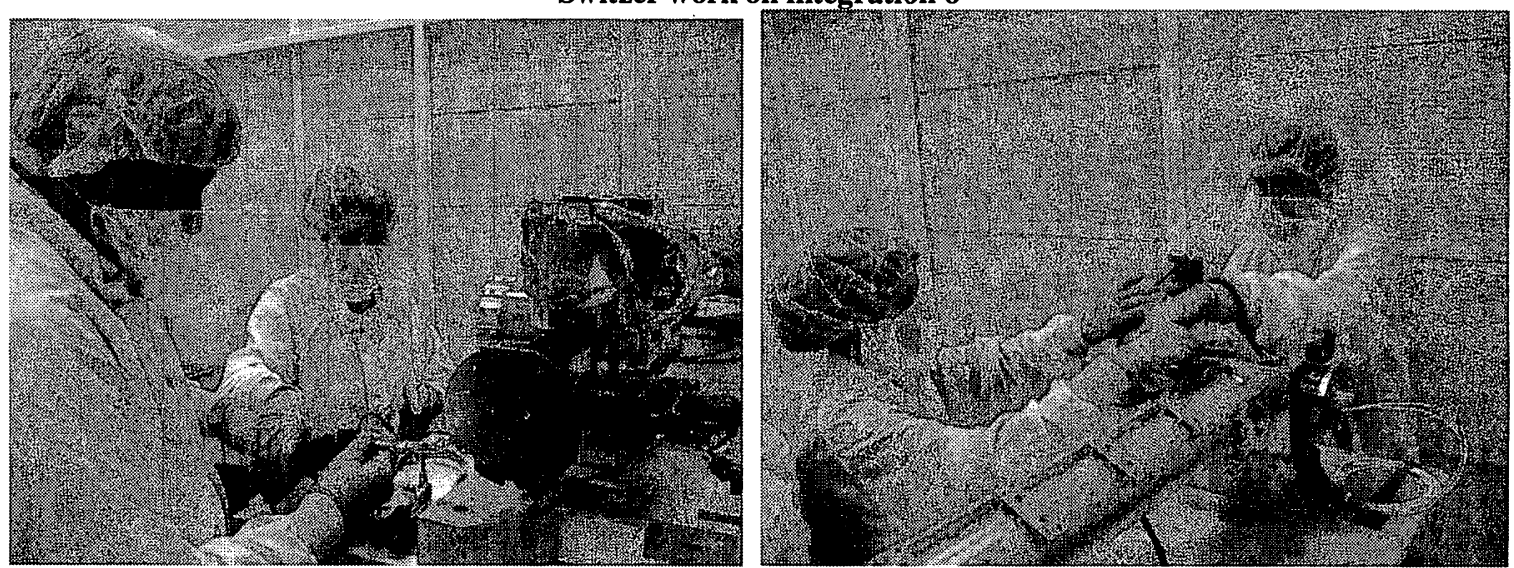

Figures 12 a) Richard Chuska and Melanie Ott conduct transmission testing on FOB 2 with gimbals now attached to the entire boom arm, b) Melanie Ott and Adam Matuzseski integrate the FOB 2 to a safe position after integration down the boom, while awaiting the mandrel.

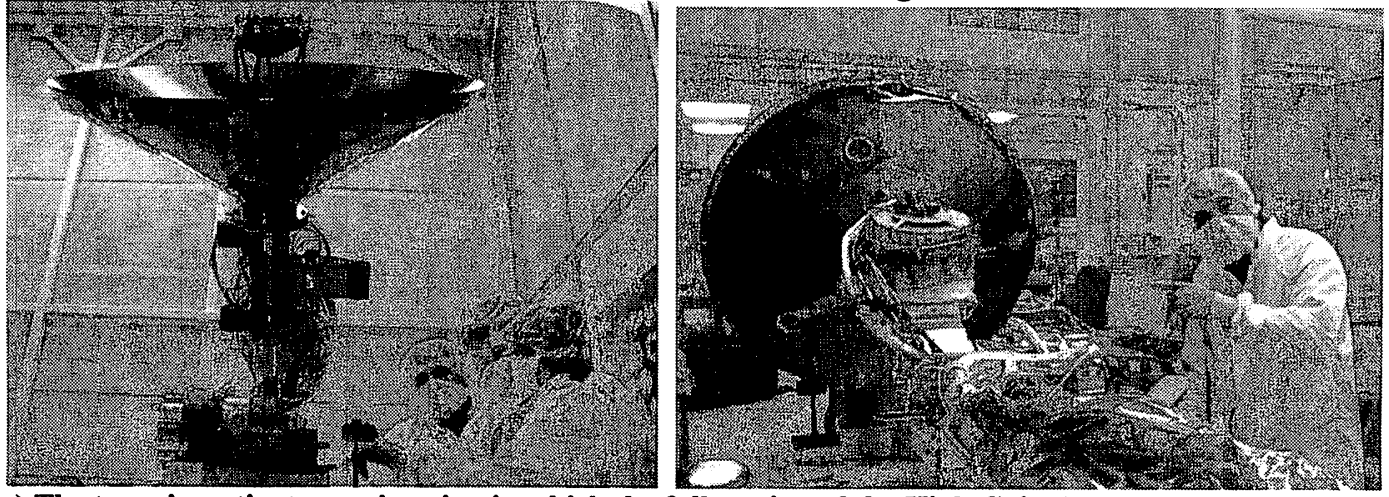

Figure 13a) The team investigates at situation in which the full motion of the High Gain Antenna comes close to the optical cable routing configuration. B) Richard Chuska motions that the High Gain Antenna is now fully integrated to the gimbals and boom arm. 

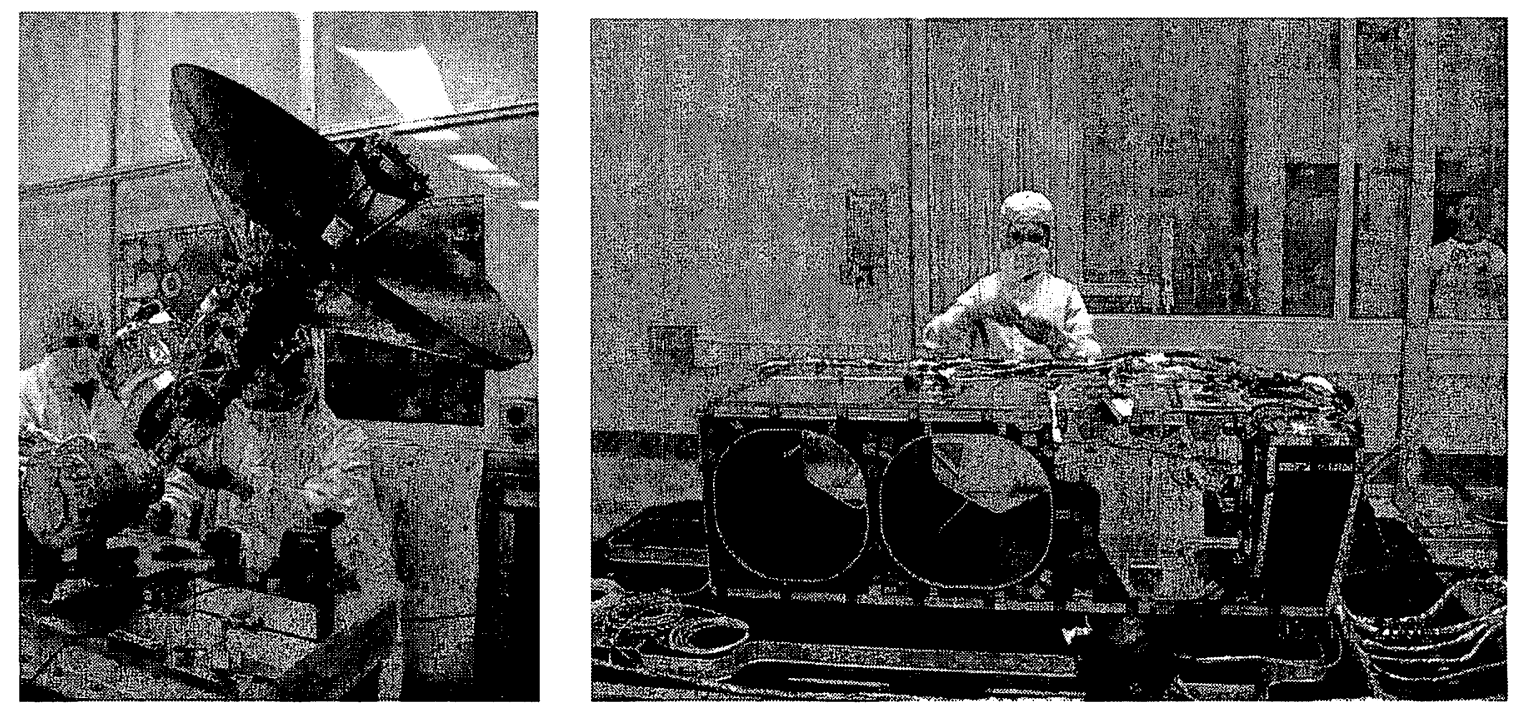

Figure 14: a) Richard Chuska, Adam Matuzseski and Melanie Ott watch the motion of the HGA with gimbals running through their full range of motion, b) Melanie Ott routing FOB 3 under the Instrument Module Deck

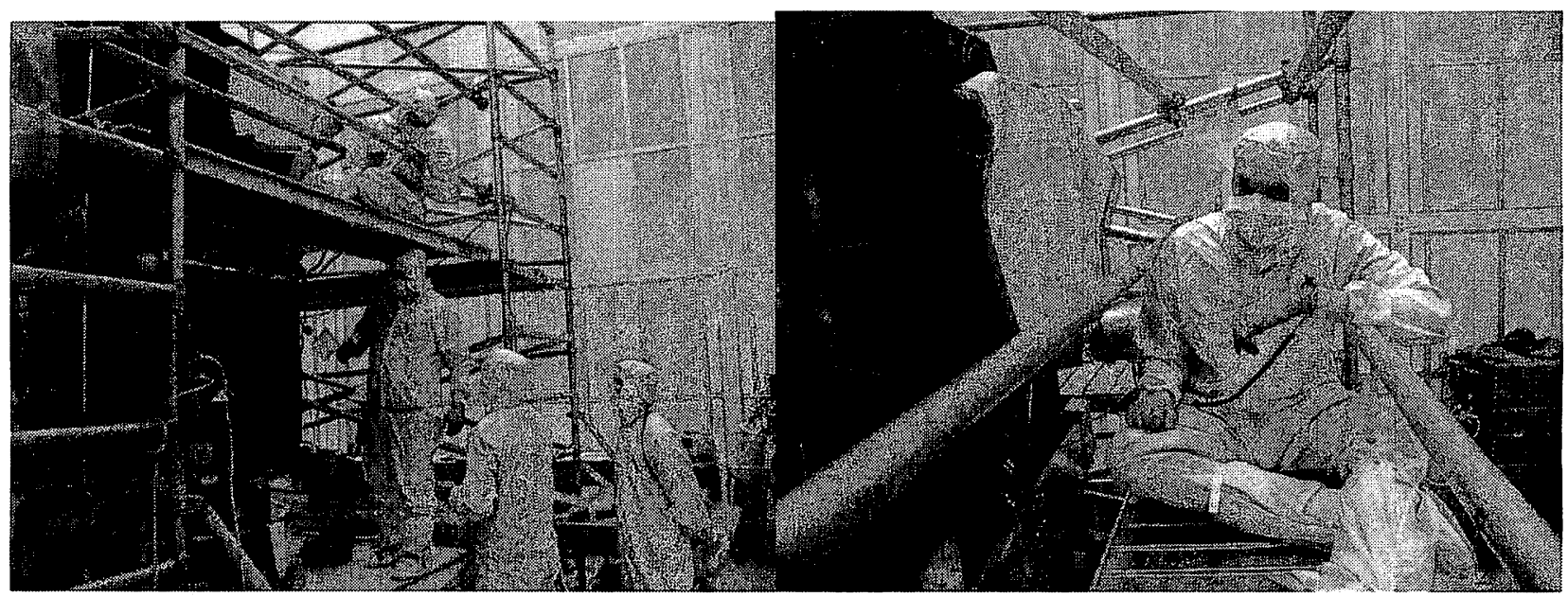

Figure 15 a) William "Joe" Thomes on second level scaffolding and Richard Chuska routing behind where HGAS will be stowed for launch, b) William Joe Thomes, performing FOB 3 routing on the early morning shift before space craft operational testing begins for the day. 


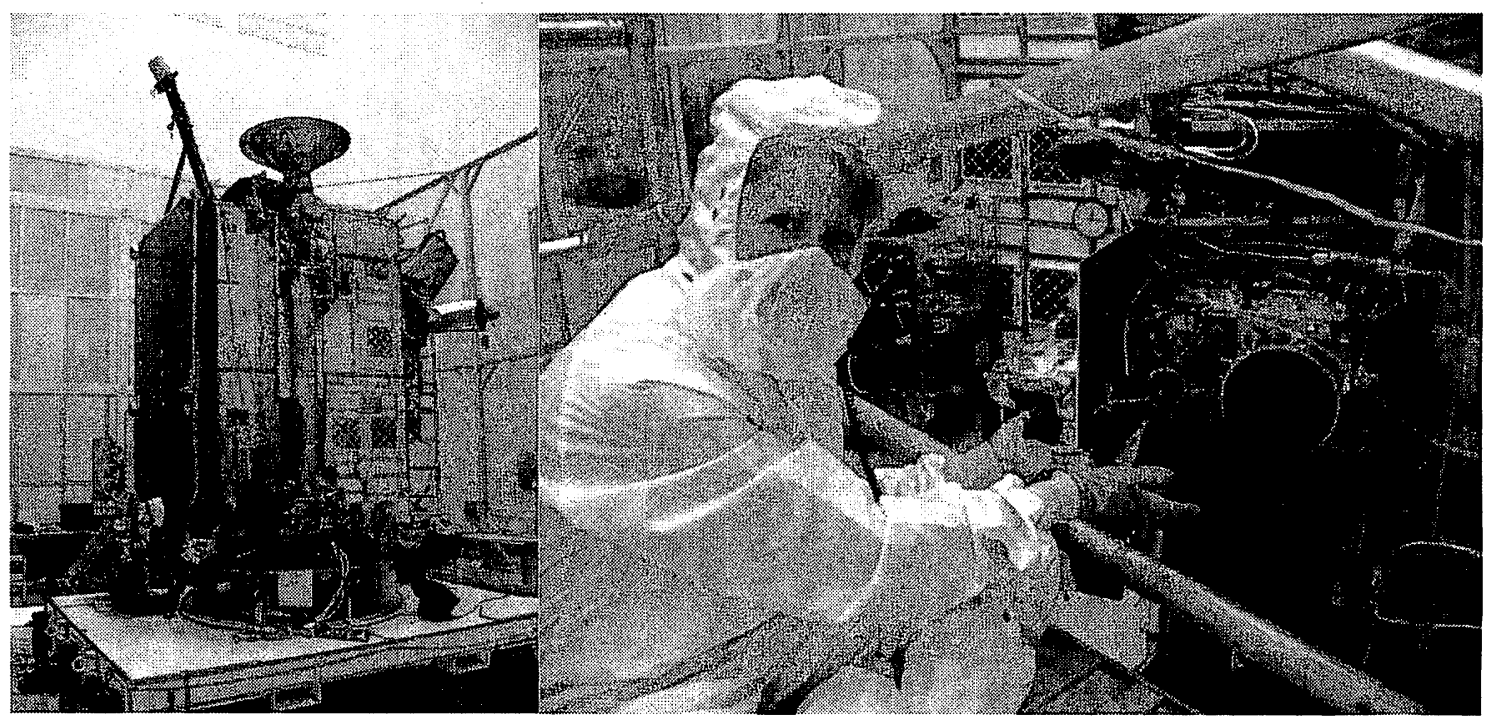

Figure 16 a) LRO fully integrated with the LRT and LOLA system, b) Melanie pointing out LOLA on LRO

\section{CONCLUSION}

Presented here was the journey of developing and integrating the optical fiber assemblies for the Lunar Reconnaissance Orbiter. The array assemblies developed for this space flight mission application are unique in the world. The LRO will be launching in late 2008 and will perform extensive mapping of the moon and the laser ranging information will help provide information to enhance the existing gravity model. For more information please consult the websites misspiggy.gsfc.nasa.gov/photonics and nepp.nasa.gov

\section{AKNOWLEDGEMENT}

The authors would like the thank the following people for their support in making this project and paper a success; Adam Matuzseski, Mark Flanegan, Michael Mulloney, Matt Showalter, Steve Schmidt, Luis Ramos-Izquierdo, John Cavanaugh, Marcellus Proctor, Jelila Mohammed, Ron Zellar, Glenn Jackson, Kusum Sahu, Henning Leidecker, Patricia Friedberg, Denise Radliff, Bruce Meinhold, Rick Jacobs, Steven Brown, Ken LaBel and Mike Sampson (NEPP program managers), Craig Tooley and Cathy Peddie (LRO Program Managers).

\section{REFERENCES}

1. Lunar Reconnaissance Orbiter Website, http://lunar.gsfc.nasa.gov.

2. Farzin Amzajerdian, "The Expanding Role of LIDAR at NASA," Photonics Spectra July 2008, pp 50 - 55.

3. D. Barry Coyle, Paul. R. Stysley, "The High Output Maximum Efficiency Resonator (HOMER) Development for Long Life, Space-Based Altimetry,” IEEE, International Aerospace Conference, Montana, March 2006.

4. X. Sun, G. A. Neumann, J. F. McGarry, T. W. Zagwodzki, J. F. Cavanaugh, J. J. Degnan, D. B. Coyle, D. R. Skillman, M. T. Zuber, D. E. Smith, "Laser Ranging between the Mercury Laser Altimeter and an Earth-based Laser Satellite Tracking Station over a 24-million-km Distance," OSA Annual Meeting, Tucson AZ, Oct. Proceedings, 2005.

5. David E. Smith, Maria T. Zuber, Xiaoli Sun, Gregory A. Neumann, John F. Cavanaugh, Jan F. McGarry, Thomas W. Zagwodzki, "Two-Way Laser Link over Interplanetary Distance," Science Magazine, Vol. 311, January 6, 2006. 
6. Melanie N. Ott, "Validation of Commercial Fiber Optic Components for Aerospace Environments," Invited paper, International Society for Optical Engineering, SPIE Conference on Smart Structures and Materials, Smart Sensor Technology and Measurement Systems, Vol. 5758, March 2005.

7. Lisa McMurray, "Tests and results of active alignment fiber optic connectors for space usage," SPIE Conference on Photonics for Space Environments IV, Vol. 2811, 1996.

8. Mark Flanegan, "Geoscience Laser Altimeter System (GLAS) Space Flight Instrument Fiber Optic System," International Society for Optical Engineering, SPIE Conference on Optical Devices for Fiber Communication II, Proceedings Vol. 4216, November 8, 2000, Boston.

9. Xiaodan "Linda" Jin, Melanie N. Ott, Frank V. LaRocca, Richard F. Chuska, Stephen M. Schmidt, Adam J. Matuzseski, Shawn L. Macmurphy, William J. Thomes, Robert C. Switzer "Space Flight Qualification on a Novel Five-fiber Array Assembly for the Lunar Orbiter Laser Altimeter (LOLA) at NASA Goddard Space Flight Center," SPIE Optics and Photonics Conference, Photonics Technology for Space Environments II, Vol. 6713, Aug 28, 2007.

10. General Environmental Verification Standard, for GSFC Flight Programs and Projects, GSFC-STD-7000, http://msc-docsrv.gsfc.nasa.gov/cmdata/170/STD/GEVS-STD-7000.pdf

11. Melanie Ott, Donald Barry Coyle, John Canham, and Henning Leidecker, "Qualification and Issues with Space Flight Laser Systems and Components," International Society for Optical Engineering Conference on Lasers and Applications in Science and Engineering, Solid State Lasers XV, Technology and Devices, SPIE Vol. 6100 January 2006.

12. David E. Smith, 19th International Laser Ranging Workshop, Canberra Australia, Oct 16-20 2006.

13. Melanie N. Ott, Xiaodan "Linda" Jin, Frank V. LaRocca, Adam J. Matzuseski, Richard F. Chuska, Shawn L. Macmurphy, "Requirements Validation Testing on the 7 Fiber Array Connector/Cable Assemblies for the Lunar Reconnaissance Orbiter (LRO)," invited paper, SPIE Optics and Photonics Conference, Photonics Technology for Space Environments II, Vol. 6713, Aug 28, 2007. 